\title{
Secure Transmission Using Bivariate Principle System for WSN
}

\author{
${ }^{* 1}$ Dr. G. Suresh, ${ }^{2}$ Dr. A. Senthil Kumar \\ 1,2 Department of CSE, Kings Engineering College, Chennai \\ Email: suresh@kingsedu.ac.in,senthilkumar@kingsedu.ac.in
}

Received: 06th April 2020, Accepted: 05th May 2020, Published: 30th June 2020

\begin{abstract}
Protecting and checking the hubs consistently is hard for enormous scale sensor systems. The expectation of this effort is to distinguish the enemy hub and to shield the information from the aggressors. To recognize the antagonist (malicious) hubs and to confine them from the information broadcasting course Binomial Principle System (BPS) technique is utilized. A copy course is produced utilizing BPS in which the source, sink, and routing hubs does not uncover their unique hub ID's and information ID's. Verified and private classified data's can be ignored the nodes by assessing grade factor and fake path even within the sight of malevolent hubs. Simulation analysis is brought out through NS2 test system device and the measurements utilized are Packet delivery proportion, lost proportion, throughput, and false recognition proportion are evaluated.
\end{abstract}

\section{Keywords}

Grade Factor, Binomial Principle System, Artificial Route, Wireless Sensor Networks.

\section{Introduction}

Sensor networks is usually made out of huge amount of sensor hubs and it has highlights like minimal effort and low force utilization, and truly perfect in size. The usefulness of sensor systems incorporates detecting of ecological information and transmitting it to the Base Station (BS) or sink hub. It executes restricted information preparing and imparts over short separations. BS comprises of sink hub that is associated with the external world.

Subsequently applications require correspondence in WSN ought to be exceptionally secure. The primary security dangers in sensor networks are unbound radio connections and traded off sensor hubs. Specific Forwarding is otherwise called as gray-hole assault. The pernicious hub prevents the particular bundles from sending it to the following hub or it just drops the parcels from them. It causes disavowal of administration for that specific hub or a gathering of hub. In warm-hole assault the messages are replayed persistently between the far off hubs prompting rushed exhaustion of their energy assets.

\section{Related Works}

Many securities related methods were proposed and some of them are discussed here. Secured position estimation [1] method was presented for verifying the area data of hubs. Secure key generation process comprises two-way validation and trivial encryption are the segments for keeping the hubs from area based assault and intermediate assault. Trust sensing based Secure Routing Mechanism (TSRM) [2] had presented with the capacity to restrict different sorts of assaults and this plan likewise have lightweight qualities at the same time. Security course determination calculation raised dependent on trust degree. Anyway it is practically difficult to distinguish the individual hub's conduct in genuine conditions. Privacy Procedure includes a Route Extrapolation (PPRE) [3] had projected to conceal the node ID pairs for offering strong fortification over powerful wide-ranging attacker.

To detect the selfishness level of the malicious nodes Trust Variable Factor (TVF) was presented [4] based on the achieved node's trust rate. By taking node's outstanding vitality level, data packets count that has sent and received from the hub, the TVF is determined. For accomplishing unknown correspondence the Anonymous Course Routing (ACR) [5] had presented. Here the hub personalities are concealed, and then the data transmitted from the hub is scrambled utilizing pair-wise key, with the goal that antagonist placed in the middle of the dispatcher and collector hub so that the adversaries placed in the middle will not able to hack the real data. Hub Uncorrelated fictitious name Pair-wise [6] scheme had presented to give hub classification adjoining uninvolved assaults happened during the correspondence among hubs. Robbering hub personality through pen name causes framework misfortune since alias leads gets derived when associated to a particular hub. Competent Anonymous Communiqué Protocol (CACP) for WSN [7] had presented to accomplish secrecies for every single hub with little outflow on calculation, stockpiling, and correspondence.

Multi-User Broadcast Authentication (MUBA) strategy [8] was proposed to diminish correspondence cost because of the transmission of open key declarations. Broadcasting verification plot dependent on identification (ID) based cryptography used to limit calculation and correspondence costs; MSBA is utilized to verify matching free ID-based mark plans with message recuperation. Blunder recuperation was executed during the 
between bunch directing so as to forestall start to finish mistake recuperation [9]. Security is accomplished by confining the dangerous hubs utilizing sink-based routing design examination.

The aim of this scheme is to recognize the hub mis-behaviour and diminish the overhead in WSN. This plan comprises of 3 stage, for example, Acknowledgment (ACK) stage, Secure Acknowledgment (S-ACK) stage and Misbehavior Verification (MV) stage. If source does not get the affirmation from the sink during ACK stage, the source sent the S-ACK packet to the S-ACK stage. S-ACK stage produces the bad conduct report. Misbehaviour Verification stage checks the trouble making report is confirmed or not [10].

\section{Proposed Method}

Secure Transmission using Bivariate Principle System (STBPS) for WSN is proposed. The principle motivation behind this examination is to classify the foe hub in the system and to disregard them from the routing way. The information is scrambled to shield from the vindictive onlooker at the sender part. Initially, the hubs present in the steering way are checked for its innovation by their solicitation preparing values utilizing Grade Factor (GF) computation. Then Bivariate Principle System (BPS) used to improve security algorithm for the proposed method. Malignant nodes couldn't detect the passing information over the route but it continuously monitors the network for the operations. Therefore, hubs can send classified message during information transmission in the system with the assistance of System Administrator in this proposed STBPS method.

\section{Identifying Customary Nodes}

Nodes in the system classified into 2 classes such as Adversary and Customary class. Adversary Nodes (AN) are selfish conduct hubs and Customary Nodes $(\mathrm{CN})$ performs typical tasks. These hubs are sorted in the interest of trust estimations of every individual hub that is resolved dependent on the evaluation count. Evaluation factor estimation of hubs incorporates their vitality level and the vitality reference level. GH hubs fall under CN and GL hubs fall under AN. GF calculation is carried out on the basis of total number of processed request and reply messages as per required data transmissions using equation 1 ,

An individual private key is shared for all the nodes based on grade factor value and the key is predistributed with the base station. GH nodes are assigned with strong private key but the malignant nodes present in the route can drop the false data to the transmitting information. Therefore artificial node ID's can be created for protecting the data from the malignant node's false injection.

Applying BPS to Nodes:

To ensure the information transmission by means of vindictive hubs, the source hub produces counterfeit course with the goal that the hand-off hub won't uncover their unique ID just as the source and goal unique ID. The hand-off hub ID is increased with bi-variate qualities then transmits recently produced hub ID for its upcoming transfer hub. 'A' can screen the information transmission yet it can't distinguish the specific source hub which correspondingly transmits the necessary data. Thus the supply hub sends private data to goal.

\section{Generating Artificial ID:}

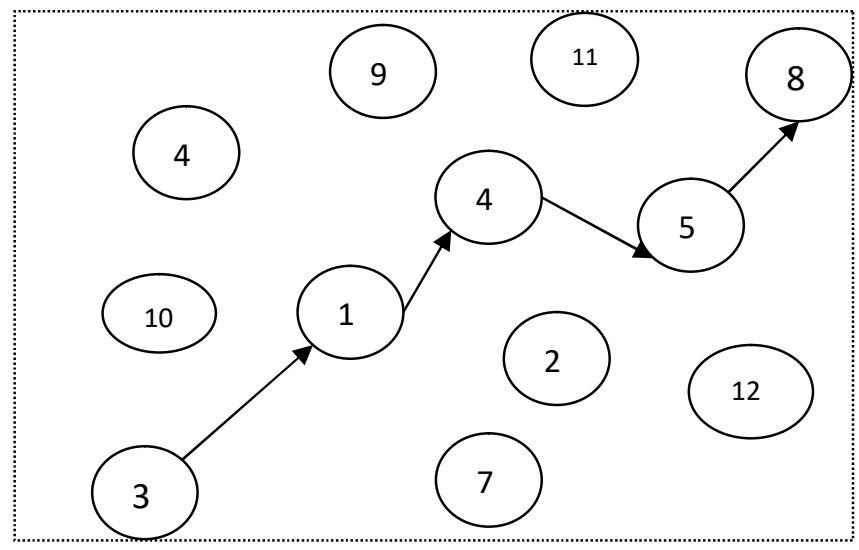

Figure 1: Artificial Route Creation

Bi-variate information is characterized as the information that possesses two factors. This information is produced by taking the connection exists among these two factors. Model, the source hub is taken as 'An' and goal hub is ' $\mathrm{B}$ ', and the midway hub check is ' $\mathrm{n}$ '. Bi-variate guideline framework is characterized using equation 2, 
$\mathrm{BPS}=(\mathrm{A}-B)^{\mathrm{n}}$

BPS is used to create artificial route is given as $(3-8) 4 \rightarrow\{34+4(33)(4)+8(32)(82)+4(3)(83)+84\} \rightarrow$ $81+432+4608+6144+4096$.

From figure.1, it is demonstrated that the source hub is 3 and goal hub is 8 and the middle of the road hubs are 1, 4 and 5. A false ID is produced for every single hub that present in the course from source to goal. False ID made for source is 16 and for sink is 4096; correspondingly false ID created for transitional (data passer) hubs is 432,4608 and 6144 . This fake course extraordinarily lessens passive assaults like gray-hole and black-hole. It likewise gives greater security to the framework. Also, artificial id created for the intermediate hubs guarantees that the dangerous onlooker cannot distinguish the hub's unique ID.

\section{Algorithm of Proposed System}

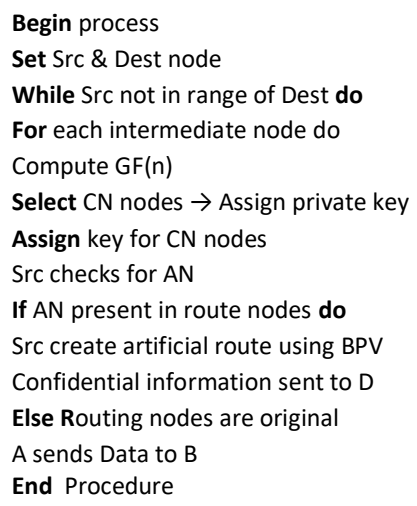

\section{Results and Discussion}

Presentation of the proposed framework is analysed by utilizing the network simulator test system. To evaluate the proposed plan 50 hubs are taken, a system in a region of $1500 \times 1500 \mathrm{~m} 2$.

Proposed system STBPS achieves good packet delivery rate compared to the TSRM the conventional method. It is clearly shown in figure 2 .

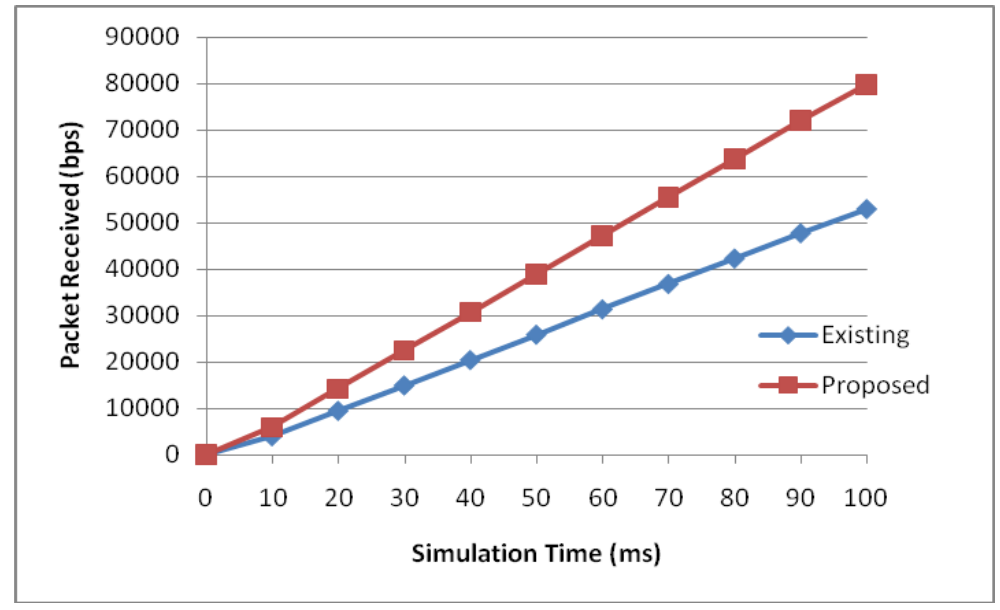

Figure 2: Packet Delivery Proportion of TSRM and STBPS 


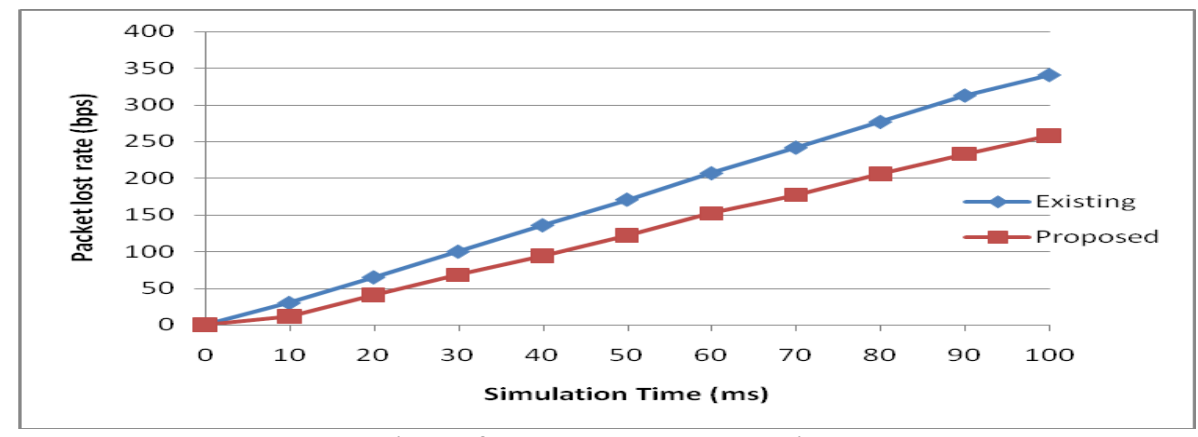

Figure 3: Packet Loss Proportion

The loss proportion is low for the proposed scheme STBPS due to avoiding false injection packets. In existing scheme packet loss rate is comparatively high and it is shown in figure 3 .

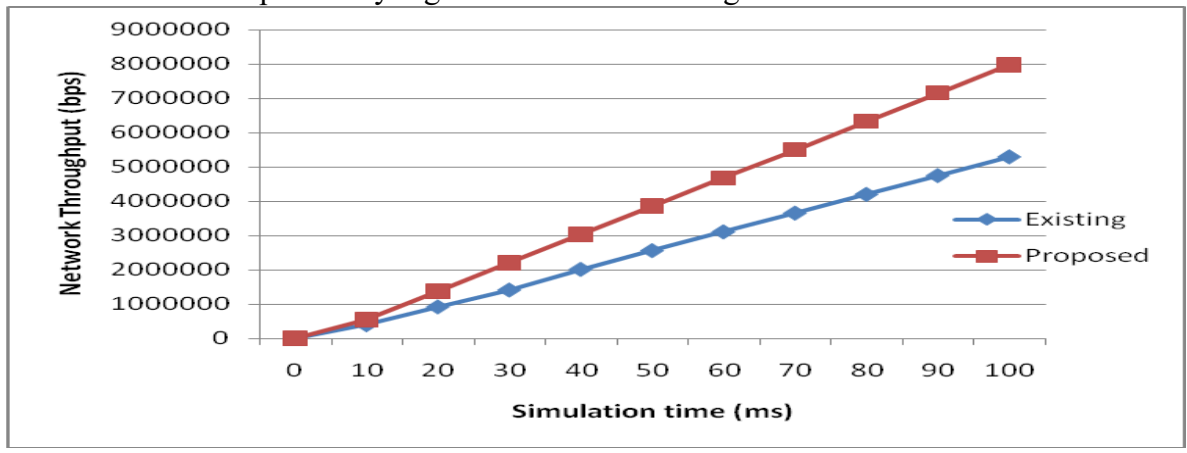

Figure 4: Throughput

Throughput is characterized as the charge point at which the information is effectively transmitted for each parcel sent. By and large the system throughput efficacy is dictated by thinking about the general precision of reachable information. STBPS proposed scheme achieves better performance over TSRM as shown in figure 4.

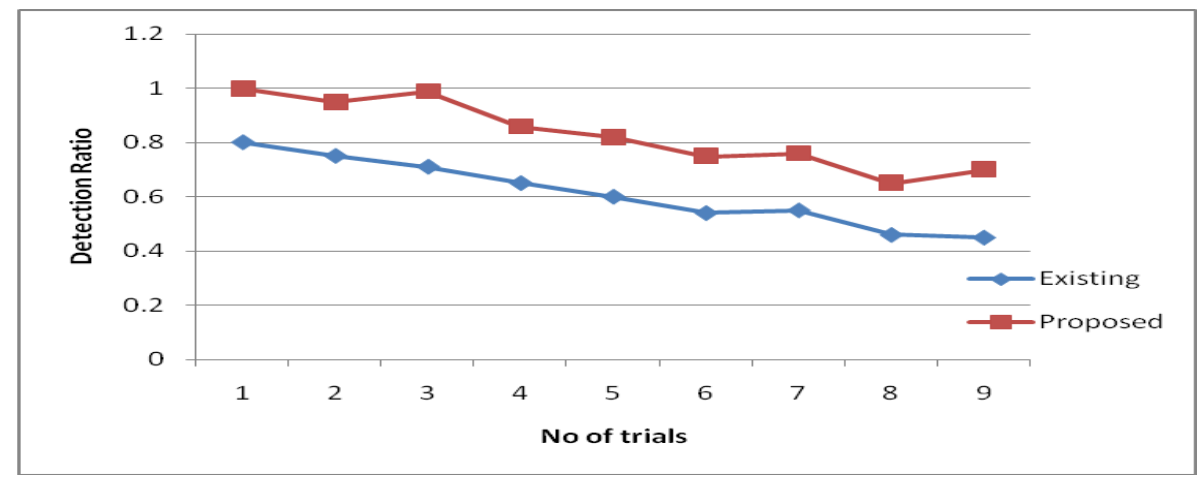

Figure 5: Detection Ratio

The false recognition proportion of nodes is distinct as the detection of adversary hubs with allusion to the customary nodes. Proposed STBPS have good ability in detecting false or adversary nodes compared to existing which is shown in figure 5.

Proposed mechanism have better false recognition rate by taking the possible number of trials compared to the existing system.

\section{Conclusion}

Secure Transmission using Bivariate Principle System for WSN is proposed to recognise the false node present in the system and to defend the valid information from the aggressors. To recognize the misbehaving hubs and to separate them from the information transmission course Binomial Principle System (BPS) method is used. Duplicate course is created utilizing BPS for all the hubs exist in the system doesn't uncover their unique hub ID's as well as information ID's. Verified and classified data's can be disregarded the hubs by assessing grade 
factor and fake course within the sight of antagonist or misbehaving hubs. Simulations results are shown to prove the network efficiency.

\section{References}

1. Jokhio, Sana H., Imran Ali Jokhio, and Andrew H. Kemp. "Light-weight framework for securitysensitive wireless sensor networks applications." IET Wireless Sensor Systems 3, no. 4 (2013): 298306.

2. Qin, Danyang, Songxiang Yang, Shuang Jia, Yan Zhang, Jingya Ma, and Qun Ding. "Research on Trust Sensing based Secure Routing Mechanism for Wireless Sensor Network." IEEE Access (2017).

3. Doomun, M. Razvi, and K. M. Soyjaudah. "Route extrapolation for source and destination camouflage in wireless ad hoc networks." arXiv preprint arXiv:1208.5569 (2012).

4. Talreja, Rahul, SriPradha Sathish, and Kamlesh Nenwani. "Trust Variable Factor: A trust based method to detect misbehaving nodes in MANET." In Electrical, Electronics, and Optimization Techniques (ICEEOT), International Conference on, pp. 3238-3241. IEEE, 2016.

5. Sheu, J-P., J-R. Jiang, and Ching Tu. "Anonymous path routing in wireless sensor networks." In Communications, 2008. ICC'08. IEEE International Conference on, pp. 2728-2734. IEEE, 2008.

6. Yang, Guang, Guining Geng, Jing Song, Zhaohui Liu, He Han, and Xiangang Gao. "A secure anonymous routing protocol in WSN." In Information and Automation (ICIA), 2013 IEEE International Conference on, pp. 415-418. IEEE, 2013.

7. Chen, Juan, Xiaojiang Du, and Binxing Fang. "An efficient anonymous communication protocol for wireless sensor networks." Wireless Communications and Mobile Computing 12, no. 14 (2012): 13021312.

8. Shim, Kyung-Ah. "BASIS: a practical multi-user broadcast authentication scheme in wireless sensor networks." IEEE Transactions on Information Forensics and Security 12, no. 7 (2017): 1545-1554.

9. Ganesh, Subramanian, and Ramachandran Amutha. "Efficient and secure routing protocol for wireless sensor networks through SNR based dynamic clustering mechanisms." Journal of Communications and Networks 15, no. 4 (2013): 422-429.

10. Kumar, A. S., \& Logashanmugam, E. (2016). Secure Acknowledgement based Misbehaviour Detection in WSN (S-ACK). Indian Journal of Science and Technology, 9(40), 96063. 